\title{
Left-Right and Upper-Lower Visual Field Asymmetries for Face Matching, Letter Naming, and Lexical Decision
}

\author{
Rogier E. Hagenbeek and Jan W. Van Strien \\ Published in Brain and Cognition 49, 34-44 (2002)
}

\begin{abstract}
The relation between left-right and upper-lower visual field (VF) asymmetries was examined for face matching, letter naming and lexical decision. Stimuli were flashed in the VF quadrants. Face matching resulted in a lower-left and upper-right VF advantage. Letter-naming resulted in a robust upper-right VF advantage. For lexical decision, no upper/lower asymmetries were found. Words were processed faster in the right than in the left VF, while nonwords were processed equally fast in both VFs. The results are discussed in terms of hypothesized structural connections of the lower versus upper visual field to dorsal versus ventral visual pathways and in terms of attentional mechanisms related to the processing of visual information in the VF quadrants.
\end{abstract}

Keywords: upper/lower visual field; left/right visual field; cerebral dominance; laterality; face perception; letter naming; lexical decision; visual pathways; visual attention

Visual half-field studies have demonstrated that tachistoscopic presentation of stimuli in the left versus right visual field (VF) results in performance asymmetries that are indicative for hemispheric specialization. In terms of stimulus or task properties, the left hemisphere primarily mediates verbal processing, while the right hemisphere mediates nonverbal, visuospatial processing. In terms of processing style, the left hemisphere is specialized in local (high spatial frequency) processing, while the right hemisphere is specialized in global (low spatial frequency) processing (e.g., Sergent, 1982).

Cohen (1982) discussed two models that explain visual half-field asymmetries, the structural model and the attentional model. The structural model explains visual half-field asymmetries in terms of the efficiency of pathways between brain areas. For instance, verbal stimuli are better recognized and processed if they are projected directly towards the left hemisphere compared to indirect connections via the right hemisphere and corpus callosum. The attentional model explains visual half-field asymmetries as a consequence of neuronal activation (Kinsbourne, 1970). For instance, the presentation of verbal stimuli results in left-hemisphere activation, which triggers a rightward attentional bias and results in a right VF advantage. Cohen (1982) has adopted a combined structural-attentional model of hemispheric asymmetries. The combined model is compatible with clinical studies, which show both structural and attentional deficits in brain-damaged patients.

In comparison to the large amount of visual laterality studies that have been conducted in healthy participants, few studies have investigated performances as a function of VF quadrant. Christman \& Niebauer (1997) presented a 
comprehensive review of the relationship between left-right and upper-lower VF asymmetries. They summarized task and input factors that have been examined with respect to both left-right and upper-lower VF asymmetries. Asymmetries in favor of the lower VF have been found for simple reaction time, global processing, and near stereoscopic vision. Asymmetries in favor of the upper VF have been found for local processing, far stereoscopic vision, visual search, and visual attention. Christman and Niebauer concluded that upper-lower VF asymmetries are at least as strong and prevalent as left-right VF asymmetries. Furthermore, they suggested a systematic link between lower and left VF processing and between upper and right VF processing. For instance, Christman (1993) reported upper and right VF advantages for local processing, and lower and left VF advantages for global processing. Likewise, Niebauer and Christman (1998) found upper and right VF advantages for categorical (above/below) judgements, and lower and left VF advantages for coordinate (near/far) judgements.

Christman and Niebauer (1997) offered tentative explanations for these links, in terms of both ecological mechanisms such as attentional biases and directional scanning habits and neural mechanisms such as the role of parvo- and magnocellular pathways and the role of dorsal and ventral pathways.

Clinical studies in patients with right-sided lesions have indicated that left VF neglect tends to be more serious in the lower than in the upper visual field (e.g., Ladavas, Carletti \& Gori, 1994). Rapcsak, Cimino and Heilman (1988) found that on a line-cancellation task, patients with neglect from right hemispheric lesions often omit more lines in the left lower than in the left upper part of the page. Furthermore, distinct neglect phenomena in the upper-lower dimension have been reported. Rapcsak et al.(1988) discussed a patient with Balint's syndrome, whose CT scan and MRI showed bilateral parieto-occipital infarctions. The left-sided lesion included most of the dominant angular gyrus (Brodmann's area 39), while the right-sided lesion included the inferior portion of the angular gyrus, the posterior portion of the middle temporal gyrus (Brodmann's area 37), and extended posteriorly into the lateral occipital gyrus (Brodmann's area 19). Although confrontation tasks showed that the patient could perceive stimuli in all four quadrants of the VF, she complained of an occasional inability to see the lower halves of images she was fixating. Cows appeared to be floating in the air without legs. On visual and tactile bisection of vertical rods, the patient demonstrated altitudinal neglect by consistently placing her mark well above the actual midpoint. Rapcsak et al.(1988) concluded that bilateral damage to the parietal lobes could lead to neglect in the lower VF.

Shelton, Bowers \& Heilman (1990) discussed a patient with bilateral inferior temporal lobe and deep occipital lobe ischaemic infarction, worse on the left, but with no evidence of parietal lobe pathology. The patient neglected far peripersonal and upper vertical space. The patient accurately localized light stimuli in the lower VF, but made systematic errors in localizing targets in the upper VF. He bisected vertical lines below the actual midpoint, in both the visual and tactile modality. On a line cancellation task, he failed to cancel targets in the upper quadrants. Although this patient had upper VF defects, this loss could not totally account for the line bisection errors in the upper VF. Shelton et al.(1990) concluded that bilateral damage to the inferior temporal lobe could lead to neglect of upper vertical space. 
Previc (1990) has hypothesized that the lower VF is involved in near visual space, while the upper VF is involved in far visual space. Near vision relies on global perceptual processing, whereas far vision relies on local perceptual processing. According to Previc, near (lower VF) versus far (upper VF) vision is disproportionately represented in the dorsal versus ventral visual pathways. Others have disputed Previc's claim of an enhanced lower VF representation in the posterior parietal cortex and an enhanced upper VF representation in the inferior temporal cortex (e.g., Bruce \& MacAvoy, 1990). Previc's hypothesis offers a structural model to explain upper versus lower VF differences, whereas the clinical data may support an attentional model. The two contrasting cases of altitudinal neglect described above suggest that the inferior temporal cortex is involved in attention to the upper $\mathrm{VF}$, whereas the posterior parietal cortex is involved in attention to the lower VF.

In the present study, we examined by means of three typical laterality tasks (a face-matching task, a letter-naming task, and a lexical decision task) whether tachistoscopic presentations in the VF quadrants provide additional information about visual-field advantages in comparison to the customary tachistoscopic left/right half-field presentations.

For face-matching, we used the cartoon drawings from the Ley and Bryden (1979) study, in which these faces yielded a left VF superiority in terms of recognition accuracy. For letter-naming, we used strings of three consonants as stimuli. These letter strings typically yield a right VF advantage (e.g., Van Strien \& Heijt, 1995; Van Strien \& Morpurgo, 1992). For the lexical decision task, we presented four-, five- and six-letter words and nonwords. For these stimuli, also a right VF advantage has been demonstrated (Van Strien \& Boon, 1997). If, as Christman and Niebauer (1997) have suggested, there is a systematic link between lower and left processing and upper and right processing, then we would expect to find a lower left VF advantage for face matching and an upperright VF advantage for letter naming and lexical decision.

\section{EXPERIMENT 1: FACE MATCHING}

\section{Method}

Participants. Twenty strongly right-handed university students (10 men, 10 women) participated. Their ages ranged from 21 to 28 years, with a mean age of 24.3 years. Strength of handedness was measured by means of a hand-preference questionnaire with 10 items. The scores could range from -10 (extremely left handed) to +10 (extremely right-handed). Participants were considered to be strongly right-handed if they had a score of +8 or more. They were paid for their participation.

Apparatus. The stimuli were presented on a Nec MultiSync 5FGe computer monitor. The participants were seated at 50 centimeters from the computer screen, with the center of the screen at eye level. Stimulus presentations and response registration were programmed using the Experimental Run Time System software package (Beringer, 1994). They responded bimanually by pressing response keys for index and middle fingers. Half of the participants (5 men, 5 women) responded with both index fingers for same decisions and with 
both middle fingers for different decisions. The other participants responded in the opposite manner.

Stimuli. The stimuli were cartoon drawings from the set of the Ley and Bryden (1979) study. Four faces with a neutral emotional expression were selected. The drawings were digitized for use on the computer. The faces were viewed frontally, presented black on white (see Fig. 1). The face-matching task consisted of 66 pairs of identical faces and 66 pairs of different faces. There were 36 practice trials and 96 trials in the experimental run (12 same and 12 different trials in each quadrant). In each quadrant, target faces were presented at an angle of approximately $1.0^{\circ}$ (inside) to $4.6^{\circ}$ (outside) left or right from the vertical midline and an angle of $1.0^{\circ}$ to $6.8^{\circ}$ above or below the horizontal midline. The probe face was always positioned in the center of the screen.
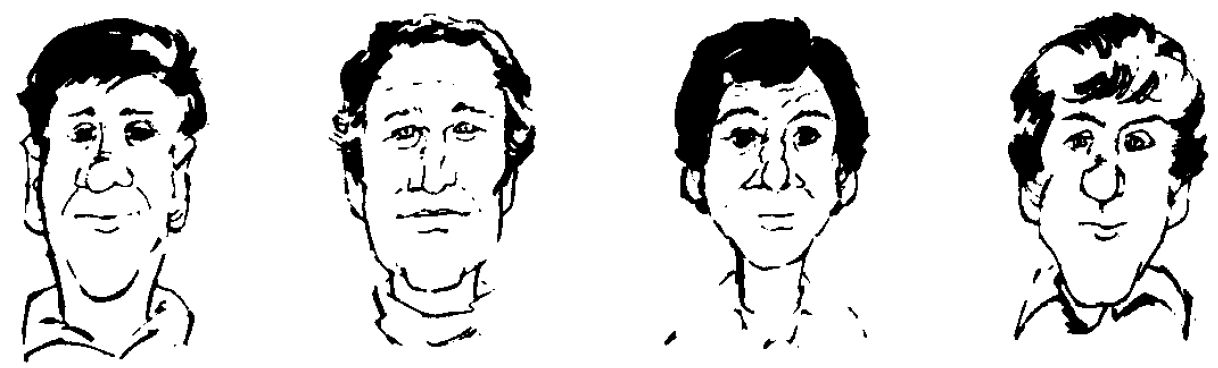

FIG. 1. The four faces used in Experiment 1 (after Ley and Bryden, 1979).

Design and Procedure. Trials were presented in a pseudorandomized order with no more than three successive presentations in the same VF quadrant and no more than three successive presentations of the same decision type (same or different). Each trial was started by the experimenter. The sequence for each experimental trial was: (a) a $500 \mathrm{~ms}$ warning tone $(1000 \mathrm{~Hz})$ followed by the $1500 \mathrm{~ms}$ presentation of the fixation cross, (b) the $150 \mathrm{~ms}$ presentation of the target face in one of the visual quadrants, (c) the $2000 \mathrm{~ms}$ presentation of the probe face in the center of the screen, and (d) the $1500 \mathrm{~ms}$ presentation of the fixation cross. The maximum RT was set at $2000 \mathrm{~ms}$. Participants had to make a same-different judgement as quickly as possible. They were explicitly instructed to fixate on the central fixation cross. They were told that because the quadrant of presentation was determined randomly, optimal performance could only be obtained by proper fixation.

\section{Results and discussion}

Latency data. For each participant, response latencies were averaged across hands and mean reaction times were computed as a function of type of response (same/different) and VF quadrant. An ANOVA, with type of response, upper/lower VF, and left/right VF as factors within-subjects and gender as a factor between-subjects, revealed a significant interaction of upper/lower and 
left/right VF, $F(1,18)=4.43, p=.05$. From table 1, it can be seen that the fastest RTs were found when faces were presented in the upper-right and lower-left VFs. In addition, a significant four-way interaction of sex, type of response, upper/lower VF, left/right VF was found, $F(1,18)=5.72, p<.03$. This interaction is displayed in Figure 2. From this figure, it can be seen that the upper-right and lower-left VF advantage is found in men for different trials, whereas it is found in women for same trials.

TABLE 1

Mean Latencies in Milliseconds (Upper Part) and Accuracy Scores (Lower Part) for Face Matching as a Function of Visual Field Quadrant

\begin{tabular}{|c|c|c|}
\hline \multicolumn{3}{|c|}{ Latency } \\
\hline & \multicolumn{2}{|c|}{ Visual Field } \\
\hline & Left & Right \\
\hline Upper visual field & 773 & 758 \\
\hline Lower visual field & 752 & 772 \\
\hline \multicolumn{3}{|c|}{ Accuracy } \\
\hline \multicolumn{3}{|c|}{ Visual Field } \\
\hline & Left & Right \\
\hline Upper visual field & $79.8 \%$ & $85.8 \%$ \\
\hline Lower visual field & $84.5 \%$ & $83.8 \%$ \\
\hline
\end{tabular}

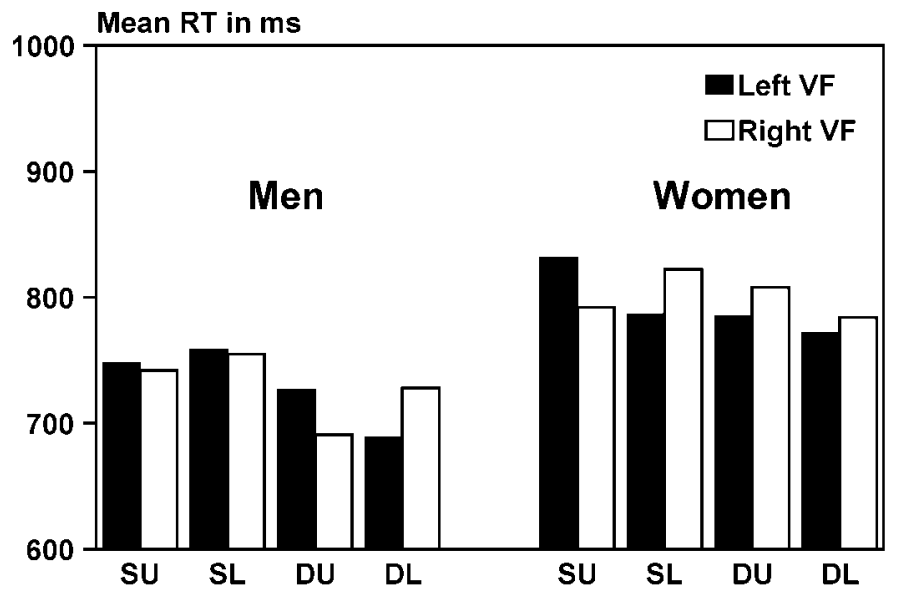

Fig. 2. Mean latencies for face matching as a function of left/right visual field, upper/lower visual field, type of respons, and sex (SU = same trials, upper visual field; SL = same trials, lower visual field; $\mathrm{DU}=$ different trials, upper visual field; $\mathrm{DL}=$ different trials, lower visual field). 
Accuracy data. The numbers of correct responses in each condition were subjected to an ANOVA. The analysis revealed a significant main effect for type of response, $F(1,18)=28.26, p<.001$, indicating that more correct responses were given when the faces were different $(M=91.6 \%$ correct) than when faces were the same $(M=75.5 \%)$. We found a significant interaction between upper/lower $\mathrm{VF}$ and left/right VF, $F(1,18)=5.20, p<.05$. From the lower part of Table 1, it can be seen that the lowest percentage of correctly identified faces was found in the upper-left VF. Furthermore, a significant interaction was found for type of response and left/right $\mathrm{VF}, F(1,18)=6.57, p<.05$. Inspection of the data revealed that identical faces were better identified in the right VF $(M=78.8 \%)$ than in the left VF $(M=72.5 \%)$, whereas different faces were processed equally accurate in the left $(M=92.1 \%)$ and the right VF $(M=91.3 \%)$.

Discussion. RTs were shorter for faces in the lower-left and upper-right VF (in men for different faces in particular, in women for identical faces in particular), and less faces were matched correctly in the upper-left quadrant than in the other quadrants. The latency data support Christman and Niebauer's (1997) suggestion of a systematic link between lower and left VF processing and between upper and right VF processing, but are contrary to our expectations of an exclusive lower-left VF advantage for face processing.

A four-way interaction revealed that the lower left and upper right VF advantages mainly pertained to different pairs in men and to same pairs in women. The reason for this sex difference is not immediately evident and needs further investigation. Most probably, men and women employ different strategies for face matching.

From the accuracy data, it was obvious that different pairs were much more accurately detected than same pairs. Our latency data revealed a nonsignificant trend with different responses $(M=748 \mathrm{~ms})$ being slightly faster than same responses $(M=780 \mathrm{~ms})$. Therefore, the higher accuracy for different pairs is not a consequence of a speed-accuracy trade-off. Apparently, same pairs were more doubtful than different pairs and our participants were biased to judge doubtful trials as different. In addition, the significant interaction of type of response and left/right VF suggests that the right hemisphere (left VF) has more difficulty in accurately recognizing same pairs than does the left hemisphere (right VF).

As the exact mechanisms to explain the diagonal VF advantage for face matching latencies remain unclear, only a tentative (post hoc) explanation can be offered. The nature of hemispheric (left-right) specialization has been described in terms of local processing in the left hemisphere vs. global processing in the right hemisphere. The cartoon faces may have been processed in a local fashion (face features processing) in the right VF (left hemisphere), and in a global fashion (whole face processing) in the left VF (right hemisphere). Because local processing is not only biased to the right VF but also to the upper VF, an upperright VF advantage may be found when faces are processed in a local manner. And because global processing is not only biased to the left VF but also to the lower VF, a lower-left VF advantage may be found when faces are processed in a global manner.

There is PET and fMRI evidence that photographs of faces activate the left and right fusiform gyri, and that this activation is more extensively on the right than on the left side (e.g., Haxby, Horwitz, Ungerleider, Maisog, Pietrini, \& Grady, 1994; Puce, Allison, Asgari, Gore, \& McCarthy, 1996). Therefore, it also might have been hypothesized that faces are better recognized in the upper VF, 
especially in the upper-left VF. In the present experiment however, neither accuracy nor latency yielded an upper-left VF advantage. In fact, the accuracy was lower in this quadrant than in the other three quadrants. It is probable that cartoon drawings activate other cortical areas than do photographs. Also, there may be processing differences between mere face perception, as in the Puce et al. (1996) fMRI study, and the present face-matching task.

\section{EXPERIMENT 2: LETTER NAMING}

\section{Method}

Participants. The same participants as in the face-matching task were administered an unilateral letter-naming task. All participants had started with the face-matching task, followed by the letter-naming task. This task order was chosen to prevent a verbal set at the beginning of the face-matching task.

Apparatus and Stimuli. For the presentation of the stimuli, the apparatus of experiment 1 was used. The stimuli were made up of sets of three consonants presented horizontally in one of the four quadrants. For the composition of each 3-letter set, the following letters were used: C,F,G,H,J,K,L,N,P,S,V,Z. The choice of letters was based on the results of a study by Schmuller (1979) indicating a low probability of confusion among these letters. Trigrams of uppercase letters were presented at an angle of approximately $3.4^{\circ}$ (inside) to $5.4^{\circ}$ (outside) left or right from the vertical midline and an angle of $2.0^{\circ}$ to $2.9^{\circ}$ above or below the horizontal midline.

There were 96 unique 3-letter sets. The participants received 24 practice trials. The experimental run consisted of 72 trials (18 in each quadrant). The trials were pseudorandomized with no more than three successive 3-letter presentations in the same VF quadrant. To avoid effects due to specific letter combinations, the letter sets were rotated by one quadrant, after every participant.

Procedure. Each trial was started by the experimenter. The sequence for each experimental trial was: (a) a $500 \mathrm{~ms}$ warning tone $(1000 \mathrm{~Hz})$ followed by the $1500 \mathrm{~ms}$ presentation of the fixation cross, (b) the $110 \mathrm{~ms}$ presentation of a 3letter set in one of the VF quadrants, (c) the $2500 \mathrm{~ms}$ presentation of the fixation cross. The participants task was to verbally report the three letters immediately after presentation. Participants were told to stay focussed on the fixation cross in order to obtain optimal performance.

\section{Results and discussion}

Because a preliminary ANOVA revealed no significant main effect or interactions related to gender, this factor was not taken into consideration in the subsequent analysis. The mean number of correctly identified letters in each quadrant is given in Table 2. Across upper and lower VFs, there was a significant right VF advantage, $F(1,19)=5.70, p<.05$, and across left and right VFs, there was a significant upper VF advantage, $F(1,19)=6.87, p<.02$. In addition, a significant interaction of both factors was found, $F(1,19)=4.52$, $p<.05$. From table 2, it can be seen that letters presented in the upper-right VF were identified better than letters presented in the other VF quadrants. The 
contrast of the upper-right quadrant versus the mean of the other three quadrants yielded a significant difference, $F(1,19)=29.42, p<.001$.

TABLE 2

Mean Number of Correctly Named Letters as a Function of Left/Right and Upper/Lower Visual Fields

\begin{tabular}{|c|c|c|c|}
\hline & \multicolumn{2}{|c|}{ Visual Field } & Total \\
\hline & Left & Right & \\
\hline Upper visual field & 40.85 & 44.10 & 84.95 \\
\hline Lower visual field & 39.95 & 41.20 & 81.15 \\
\hline Total & 80.80 & 85.30 & 166.10 \\
\hline
\end{tabular}

In sum, we found both a right VF advantage and an upper VF advantage for letter naming. Apparently, these two advantages are additive, leading to a superior upper-right VF performance in particular. The robust upper-right VF advantage is in accordance with our expectations and with Christman and Niebauer's (1997) suggestion of a systematic link between upper and right VF processing. There may be an anatomical basis for the superior upper-right processing of letter strings. Using fMRI, Puce et al. (1996) demonstrated strong activation of the left occipitotemporal and inferior occipital sulci in response to centrally presented letter strings. In contrast to our participants, the participants in the Puce et al. study did not have to name the letters. As Puce et al. have noted, their results concerned prelexical letter processing. Nevertheless, the right-upper VF advantage for letter-naming may be a consequence of the more efficient processing of letter strings by the left occipitotemporal and inferior occipital cortex. To examine whether later stages of word processing are related to VF quadrant effects, we employed a lexical decision task in the third experiment.

\section{EXPERIMENT 3: LATERAL DECISION TASK}

\section{Method}

Participants. For the third experiment, new participants were recruited. Sixteen strongly right-handed male university students with a score of +8 or more on the 10-item handednes questionnaire were selected and were paid for their participation. Their ages ranged from 19 to 27 years, with a mean age of 23.8 years.

Apparatus and Stimuli. The apparatus of the previous experiments was used. There were 96 experimental trials ( 12 words, 12 nonwords in each quadrant). Words and nonwords consisted of four, five, or six uppercase letters. Nonwords were constructed by changing one or two letters in a four-letter word, two letters in a five-letter word and two or three letters in a six-letter word. Furthermore, words and nonwords were also matched for length, both within and between each VF quadrant. Words in each quadrant were also matched for word frequency and imageability. Words and nonwords subtended $1.6^{\circ}$ (four-letter stimuli) to $2.6^{\circ}$ (six-letter words) of visual angle 
horizontally. The stimuli were presented at an angle (measured at the middle of the word) of approximately $2.2^{\circ}$ left or right from the vertical midline and an angle of $1.4^{\circ}$ above or below the vertical midline.

Design and Procedure. The stimuli were pseudorandomized with no more than three successive presentations of either words or nonwords and no more than three successive presentations in the same VF quadrant. To avoid a possible effect due to a specific set of words and nonwords, the sets were rotated by one quadrant after every participant. Each trial was started by the experimenter. The sequence for each experimental trial was: (a) a $500 \mathrm{~ms}$ warning tone $(1000 \mathrm{~Hz}$ ) followed by (b) a $1000 \mathrm{~ms}$ presentation of a fixation cross, (c) a $180 \mathrm{~ms}$ presentation of either a word or nonword and (d) the $3000 \mathrm{~ms}$ presentation of the fixation cross. The maximum RT was set at $2000 \mathrm{~ms}$. The participants were instructed to decide as quickly as possible whether the presented stimulus was a word or nonword. They were told that the best results were obtained when looking at the fixation cross in the center of the screen. Responses were given bimanually by pressing response keys for index and middle fingers. Half of the participants responded with both index fingers for word decisions and with both middle fingers for nonword decisions. The other participants responded in the opposite manner. Prior to the experimental run, the participants received 50 practice trials with another set of words and nonwords.

TABLE 3

Mean Latencies in Milliseconds for Lexical Decision as a Function of Type of Response and Visual Field Quadrant

\begin{tabular}{|c|c|c|}
\hline \multicolumn{3}{|c|}{ Latency } \\
\hline & \multicolumn{2}{|c|}{ Visual Field } \\
\hline & Left & Right \\
\hline \multicolumn{3}{|l|}{ Word } \\
\hline Upper visual field & 836 & 802 \\
\hline Lower visual field & 837 & 817 \\
\hline Total & 836 & 809 \\
\hline \multicolumn{3}{|l|}{ Nonword } \\
\hline Upper visual field & 878 & 883 \\
\hline Lower visual field & 873 & 876 \\
\hline Total & 876 & 880 \\
\hline
\end{tabular}

\section{Results and discussion}

Latency data. For each participant, mean RTs were computed as a function of type of response (word/nonword) and position (VF quadrants). The mean latencies are given in Table 3 . The analysis of variance was performed with type of response, left/right VF, and upper/lower VF as factors within-subjects. There was a significant main effect for type of response, $F(1,15)=11.38 p<.01$. Words $(M=823 \mathrm{~ms})$ were recognized significantly faster than nonwords $(M=878 \mathrm{~ms})$. Furthermore, there was a significant interaction of type of response and left/right $\mathrm{VF}, F(1,15)=5.49 \mathrm{p}<.05$, indicating that latencies to words presented in the right VF were shorter than latencies to words presented in the left VF, whereas nonwords did not yield VF differences.

Accuracy data. There was a significant main effect for type of response, $F(1,15)=17.27, p<.001$, indicating that nonwords $(91.4 \%$ correct) were identified more accurately than words $(84.9 \%$ correct). 
Discussion. Latencies were shorter to words than to nonwords. This is in agreement with previous lexical decision studies (e.g., Van Strien \& Boon, 1997). Existing words can be matched by a rapid matching of the visual word image, whereas nonwords must be processed by a slower, sequential phonological system before they can be semantically processed. There was a speed-accuracy trade-off with slower RTs but higher accuracy to nonwords compared to words. Most probably, participants, when in doubt, were biased to judge a trial as a nonword. Words were processed faster in the right than in the left VF, while nonwords were processed equally fast in both the left and right VF. Although the fastest mean RT was found for words in the upper-right VF (see Table 3), there was, contrary to our expectations, no statistically significant interaction of upper/lower and left/right VF.

Using event-related fMRI, Jessen et al. (1999) had participants indicate manually whether a letter string was a word or a unpronounceable nonword. The nonwords resulted in an activation of the classical language areas in the left hemisphere, reflecting lexical evaluation of the meaningless stimuli. Words activated the left angular gyrus and the left posterior cingulate gyrus, which, according to Jessen et al., may reflect semantic processing. The left angular gyrus is thought to play an important role in visual word recognition. It is clear that in the later stages of word processing, many areas of the left cerebral hemisphere are involved, both ventral and dorsal. It may be for that reason, that only lateral but no upper/lower VF asymmetries were found for lexical decision.

\section{GENERAL DISCUSSION}

The results of the present experiments demonstrate that VF quadrant studies reveal more information than do lateral (left/right) VF studies. Had the facematching task been a lateral VF study, we probably would have found no VF asymmetry, and we would have concluded that we could not demonstrate hemispheric specialization for face-matching. The present VF quadrant study however, demonstrates an upper-right and lower-left VF advantage, allowing us to speculate about left hemispheric local and right hemispheric global processing of faces. Likewise, divided VF studies typically demonstrate a right VF advantage for letter naming. The present VF quadrant study reveals that this right $\mathrm{VF}$ advantage is manifest in the upper VF in particular. On the other hand, the right VF advantage for lexical decision is not influenced by the upper/lower dimension.

Like lateral VF asymmetries, VF quadrant differences may be explained by both structural and attentional mechanisms. A structural model can explain the VF quadrant asymmetries in terms of the specific cortical regions to which the different quadrants are projecting. The left and right VF project toward the contralateral hemisphere. The upper and lower VF project below and above the sulcus calcarinus in V1, respectively. There is evidence that ventral and dorsal regions in V1 project preferentially to the ventral and dorsal visual pathways, respectively (Previc, 1990; Van Essen, Newsome, \& Bixby, 1986). We found a robust upper-right VF advantage for letter naming. The upper-right VF probably projects through the ventral pathway to the left temporal areas. The left occipitotemporal and inferior occipital sulci appear to be strongly involved in prelexical letter processing (Puce et al. 1996). Hence, letters may be processed 
more accurately in the upper-right quadrant than in the three other quadrants. The later stages of lexical processing, require larger areas (both dorsal and ventral)of the left hemisphere. For that reason, only left-right, but no upperlower performance asymmetries were found for lexical decision. The results for face matching are not easily explained by structural mechanisms. Neural imaging has shown that the right fusiform gyri are more involved in face processing than are the left fusiform gyri (Haxby et al., 1994; Puce et al., 1996), which would suggest a upper-left VF advantage. We found however an advantage for the lower-left and upper-right diagonal, an outcome consistent with Christman and Niebauer's (1997) conclusion of a systematic link between lower and left VF processing and between upper and right VF processing.

Attentional factors may contribute to upper-right VF advantage for face recognition and letter naming. Christman and Niebauer (1997) discussed the influences of attentional biases and scanning habits. There is evidence for an attentional bias to the upper VF. This bias has an ecological origin, as the organism is supposed to be engaged in visual search for objects that are relatively far away and hence in the upper VF. The upper VF attentional bias may be intrinsically related to structural mechanisms and hence attentional and structural explanantions need not to be mutually exclusive.

The use of a vocal response in the letter naming task may have induced an overall left-hemispheric activation, resulting in a righward attentional bias (Kinsbourne, 1970). Also, Reading habits (for left-to-right languages) may have induced a non-ecological attentional bias to the right VF. It is noteworthy however that the right VF advantage for letter naming is much more pronounced in the upper than in the lower VF, and this difference cannot be explained by left-to-right scanning habits.

Scanning habits may also have played a role in the right VF advantage for lexical decision. From the brain asymmetry literature however, there is ample evidence that these scanning habits alone cannot account for the left versus right VF differences (e.g., Bradshaw, Nettleton, \& Taylor, 1981). Nevertheless, it will be worthwhile to examine the role of scanning habits in future VF quadrant studies, for instance by presenting vertical letter arrays or words.

To further clarify the 'retinotopy' of the ventral versus dorsal visual pathways, future tachistoscopic VF research should examine performance asymmetries on what and on where tasks by means of VF quadrant rather than visual half-field studies.

\section{REFERENCES}

Beringer, J. (1994). ERTS: A flexible software tool for developing and running reaction time experiments on IBM Pcs. Behavior Research Methods, Instruments \& Computers, 26, 368.

Bradshaw, J.L., Nettleton, N.C., \& Taylor, M.J. (1981). The use of laterally presented words in research into cerebral asymmetry: Is directional scanning likely to be a source of artifact? Brain and Language, 14, 1-14.

Bruce, C.J., \& MacAvoy, M.G. (1990). Response field biases in parietal, temporal, and frontal lobe visual areas. Behavioral \& Brain Sciences, 13, 546-547.

Christman, S. D. (1993). Local-global processing in the upper versus lower visual fields. Bulletin of the Psychonomic Society, 31, 275-278. 
Christman, S. D., \& Niebauer, C. L. (1997). The relation between left-right and upper-lower visual field differences. In S. Christman (Ed.), Cerebral asymmetries in sensory and perceptual processing. Amsterdam: Elsevier.

Cohen, G. (1982). Theoretical interpretation of lateral asymmetries. In J.G. Beaumont (Ed.), Divided visual field studies of cerebral organisation. London: Academic Press.

Haxby, J.V., Horwitz, B., Ungerleider, L.G., Maisog, J.M., Pietrini, P., \& Grady, C.L. (1994). The functional organization of human extrastriate cortex: A PET-rCBF study of selective attention to faces and locations. The Journal of Neuroscience, 14, 6336-6353.

Jessen, F., Erb, M., Klose, U., Lotze, M., Grodd, W., \& Heun, R. (1999). Activation of human language processing brain regions after the presentation of random letter strings demonstrated with event-related functional magnetic resonance imaging. Neuroscience Letters, 270, 13-16.

Kinsbourne, M. (1970). The cerebral basis of lateral asymmetries in attention. Acta Psychologica, 33, 193-201.

Ladavas, E., Carletti, M., \& Gori, G. (1994). Automatic and involuntary orienting of attention in patients with visual neglect: Horizontal and vertical dimensions. Neuropsychologia, 32, 1195-1208.

Ley, R. G., \& Bryden, M. P. (1979). Hemispheric differences in processing emotions and faces. Brain and Language, 7, 127-138.

Niebauer, C. L., \& Christman, S. D. (1998). Upper and lower visual field differences in categorical and coordinate judgments. Psychonomic Bulletin \& Review, 5, 147-151.

Previc, F. H. (1990). Functional specialization in the lower and upper visual fields in humans: Its ecological origins and neurophysiological implications. Behavioral \& Brain Sciences, 13, 519-575.

Puce, A., Allison, T., Asgari, M., Gore, J. C., McCarthy, G. (1996). Differential sensitivity of human visual cortex to faces, letterstrings, and textures: a functional magnetic resonance imaging study. The Journal of Neuroscience, 16, 5205-5215.

Rapcsak, S. Z., Cimino, C. R., \& Heilman, K. M. (1988). Altitudinal neglect. Neurology, 38, 277-281.

Schmuller, J. (1979). Hemispheric asymmetry for alphabetic identification: Scaling analysis. Brain and Language, 8, 263-274.

Sergent, J. (1982). The cerebral balance of power: Confrontation or cooperation? Journal of Experimental Psychology: Human Perception \& Performance, 8, 253-272.

Shelton, P. A., Bowers, D., \& Heilman, K. M. (1990). Peripersonal and vertical neglect. Brain, 113, 191-205.

Van Essen, D. C., Newsome, J. H. R., Bixby, J. L. (1986). The projections from striate cortex (V1) to areas V2 and V3 in the Macaque monkey: Asymmetries, areal boundaries, and patchy connections. The Journal of Comparative Neurology, 224, 451-480.

Van Strien, J.W., \& Boon, C.A. (1997). Altered visual field asymmetry for lexical decision as a result of concurrent presentation of music fragments of different emotional valences. Journal of the International Neuropsychological Society, 3, 473-479.

Van Strien, J. W., \& Heijt, R. (1995). Altered visual field asymmetries for letter naming and matching as a result of concurrent presentation of threatening and non-threatening words. Brain and Cognition, 29, 187-203.

Van Strien, J. W., \& Morpurgo, M. (1992). Opposite hemispheric activations as a result of emotionally threatening and non-threatening words. Neuropsychologia, 30, 845-848. 